\title{
Effects of Post-Isometric Relaxation on Ankle Plantarflexion and Timed Flutter Kick in Pediatric Competitive Swimmers
}

Lauren Noto-Bell, DO; Brittany N. Vogel, DO; Danielle E. Senn, DO

From the Department of

Osteopathic Manipulative Medicine (Dr Noto-Bell) and

the Doctor of Osteopathic Medicine Program (Drs Vogel and Senn) at the Philadelphia

College of Osteopathic

Medicine in Pennsylvania.

Financial Disclosures: None reported.

Support: None reported.

Addresss correspondence to

Lauren Noto-Bell, DO,

Philadelphia College of

Osteopathic Medicine, 4190 City Ave, Rowland Hall,

Suite 330 , Philadelphia,

PA 19131-1694.

Email: laurenno@pcom.edu

Submitted

July 24, 2018;

revision received

October 2, 2018;

accepted

October 10, 2018.
Context: Ankle plantarflexion is thought to play an important role in swimming performance; thus, coaches and swimmers often seek ways to increase range of motion $(\mathrm{ROM})$ in the ankles.

Objective: To assess whether osteopathic manipulative treatment (OMT), specifically applying the muscle energy technique (MET) principle of post-isometric relaxation, increases ankle plantarflexion and therefore improves swimming performance.

Methods: Healthy young male and female competitive swimmers were randomly assigned to either a control, sham, or MET group. At baseline, ankle plantarflexion was measured via goniometer, and a 25-yard flutter kick swim with a kickboard was timed. After receiving the ascribed intervention, the ankle plantarflexion measurements and timed flutter kick were repeated. The initial plantarflexion measurement was retrospectively used to determine the presence of somatic dysfunction, by way of restricted motion, with reference to expected normal ranges based on age and gender. Paired $t$ tests were used to analyze the pre- to postintervention changes in ROM and flutter kick speed within each group.

Results: Fifty-five swimmers ( 32 girls and 23 boys; mean age, 12 years) participated in this study. Sixteen participants were in the control group, 17 in the sham group, and 22 in the MET intervention group. Among participants with restricted ROM, those in the MET group showed a statistically significant increase in ankle plantarflexion for the left and right ankles ( $P=.041$ and $P=.011$, respectively). There was no significant difference in ROM of the control or sham groups. For flutter kick speed, there was no significant pre- to postintervention difference in any group.

Conclusion: Although a single application of MET, using post-isometric relaxation, on participants with restricted ROM immediately significantly increased swimmers' ROM for bilateral ankle plantarflexion, it did not immediately improve their swimming performance.

J Am Osteopath Assoc. 2019;119(9):569-577

doi:10.7556/jaoa.2019.100

Keywords: ankle plantarflexion, range of motion, sports medicine

inners of swimming events are sometimes decided by mere fractions of a second. Thus, coaches and swimmers seek opportunities to maximize efficiency and minimize potential barriers to improved speed. One area that coaches and swimmers seek to improve is ankle flexibility, especially plantarflexion. Various modalities of stretching, including static, dynamic, and proprioceptive 
neuromuscular facilitation, are used in the athletic environment. ${ }^{1,2}$ However, our search of the literature in May 2016 (PubMed and EBSCOhost) and Feb 2017 (Osteopathic Medicine Digital Repository) uncovered no studies that examined the use of osteopathic manipulative treatment (OMT), or specifically muscle energy technique (MET), as a tool for effectively increasing the ankle's plantar flexion range of motion (ROM) in swimmers. Furthermore, we discovered no studies that examined the effect of MET on swimming performance.

The front crawl (or freestyle) stroke in swimming uses a flutter kick in the lower extremities. The flutter kick consists of 2 alternating motions: the upbeat kick and the integral downbeat kick. The upbeat kick functions mainly as a stabilizer of the body, while the downbeat kick generates propulsion through the water. ${ }^{3}$ In the downbeat kick, the hip slightly flexes, the knee extends, and the ankle plantarflexes. The opposite then occurs during the upbeat kick.

Optimal swimming technique is determined to a great extent by the flexibility of the ankles. ${ }^{4}$ Greater ankle plantarflexion is associated with less resistance on the downbeat kick, producing more propulsion with less effort, ${ }^{5,6}$ and freestyle kick performance depends on ankle joint flexibility. ${ }^{7}$ When the ability to plantarflex during the downbeat kick is restricted, drag is increased and less water is displaced backward, resulting in less forward propulsion. ${ }^{8}$

Muscle energy technique has been shown to increase the ROM in multiple joints. ${ }^{9-11}$ In the present pilot study, we tested the hypotheses that MET, using the principle of post-isometric relaxation (PIR), will increase ankle plantarflexion and result in faster swimming speed using the flutter kick.

\section{Methods}

\section{Participants}

Competitive swimmers, both male and female between the ages of 8 and 17 years, were recruited from Pittsburgh-area swim teams via an email advertisement. Two weeks before the study, the swim coaches distributed a handout prepared by the investigators to parents during practice. One week before the study, an email prepared by the investigators was sent by the coaches to the email addresses of the children on their listserves. The email had consent and asset forms attached; these forms were also available in hard copy at the time of the study. To ensure a safe rehabilitation window and to allay concerns of potential harm, swimmers were excluded if they had a history of ankle sprain in the previous 2 months, ankle fracture in the previous 3 months, or any previous ankle fusion surgery. Parents or legal guardians were required to sign an informed consent form, and each child was required to sign an assent statement, as stipulated by the study protocol, which was approved by the institutional review board at the Philadelphia College of Osteopathic Medicine.

\section{Experimental Protocol}

This pilot study was prospective, randomized, controlled, and partially double-blinded. Each participant was excused by the coach from a portion of his or her regular swim practice to participate in the study, which took about 10 to 20 minutes. Participants were randomly assigned to 1 of 3 groups: control, sham, or MET. Each participant attended 1 study session.

Throughout the study, investigator 1 (D.E.S.) recorded the demographics, performed and documented the ankle measurements, documented the flutter kick times as reported to her, and performed the assigned intervention. This investigator was blinded as to which participants would be determined to have restricted ROM. Investigator 2 (B.N.V.) timed the kicks and reported them; she was unaware of the groups to which each participant had been assigned or the measurements obtained by investigator 1 .

The protocol described in the following paragraphs was followed for all participants.

\section{Baseline Ankle Measurements}

To standardize the starting point of the ankles, each participant sat on the floor with his or her legs straight out 
in front and with toes pointing straight up to the ceiling. A goniometer was placed against the skin, and measurement proceeded according to the Range of Movement Guide-Ankle Injury Management trial. ${ }^{12,13}$ Starting with the left foot, the axis of rotation of the goniometer was placed $1.5 \mathrm{~cm}$ inferior to the lateral malleolus. The stationary arm of the goniometer was placed along the fibula, lining up with the fibular head. The movable arm of the goniometer was placed along the fifth metatarsal aspect of the foot. The participant was then asked to plantarflex the ankle, pointing the toes as much as he or she comfortably could, and the measurement was recorded in degrees (Figure). The same procedure was repeated with the right ankle.

\section{Initial Timed 25-Yard Flutter Kick}

To eliminate the variable of arm strokes, the participant's hands grasped a floatation board ("kickboard") held in front of the head with straight arms. Each participant was instructed to push off the wall and flutter kick one length of the pool (25 yards) as quickly as possible. This flutter kick swim was timed with a stopwatch to one-hundredth of a second (during data analysis, means were rounded to one-thousandth of a second). Timing began when the swimmer's feet left the wall and ended when the front of the kickboard contacted the opposite wall.

\section{Intervention Protocol}

The participant then underwent the specific intervention protocol for his or her group.

- Control: This group sat with the investigator for 1 minute.

- Sham: The investigator gently plantarflexed the left ankle, taking care not to approach the edge of a restrictive barrier. No muscle energy was performed. The foot/ankle was simply held in position for 3 seconds. This process was repeated 3 times. The same procedure was performed on the right ankle.
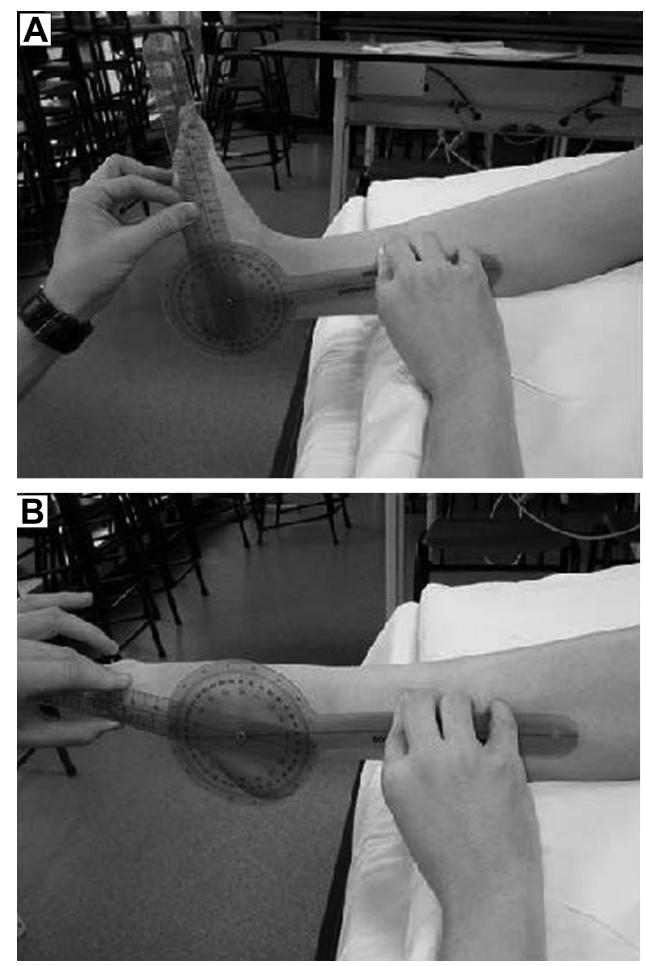

Figure

To measure ankle range of motion, (A) the participant sat on the floor with his or her legs straight out and with toes pointing straight up to the ceiling. The axis of rotation of the goniometer was placed $1.5 \mathrm{~cm}$ inferior to the lateral malleolus. The stationary arm of the goniometer was placed along the fibula, lining up with the fibular head. The movable arm of the goniometer was placed along the fifth metatarsal aspect of the foot. (B) The participant then plantarflexed the ankle, pointing the toes as much as he or she comfortably could, and the measurement was recorded.

- MET: The investigator gently plantarflexed the left ankle to the edge of the palpable restrictive barrier, and MET, using PIR, was performed by asking the participant to dorsiflex his or her foot into the investigator's hand, which applied an equal counterforce, so that no distance was actually moved by the foot. This force was held for 3 seconds, and the participant was told to relax so that the investigator could passively take the ankle to its new plantarflexion restrictive barrier. This process was repeated 3 times. The same procedure was performed on the right ankle. 


\section{After the Intervention}

Postintervention ankle measurements were obtained directly after the intervention using the same procedure previously described. The participant then went directly back to the pool for a postintervention, timed 25-yard flutter kick.

\section{Diagnosis of Somatic Dysfunction}

According to the Glossary of Osteopathic Terminology, ${ }^{14}$ a diagnosis of somatic dysfunction may be made using the following criteria: tissue texture abnormality, asymmetry, restriction of motion, or tenderness. The most objective of these criteria is restriction of motion.

Soucie et $\mathrm{al}^{15}$ provided reference values for normal joint ROM for both genders and across the lifespan. For ankle plantarflexion in their study, females aged 2 to 8 years had normal ROM from $64.8^{\circ}$ to $69.4^{\circ}$ and those aged 9 to 19 years had normal ROM from $54.8^{\circ}$ to $59.8^{\circ}$. By contrast, males aged 2 to 8 years had normal ROM from $54.4^{\circ}$ to $57.2^{\circ}$ and those age 9 to 19 years had normal ROM from $50.8^{\circ}$ to $54.8^{\circ}{ }^{15} \mathrm{We}$ used this information to retrospectively review the data collected in the present study to determine which participants had at least 1 ankle that was below the normal ROM in plantarflexion on initial measurements. These participants, therefore, satisfied the criteria for somatic dysfunction by restricted ROM. If any of the participants, on review of the initial measurements, had bilateral ankles that were within or above the normal ROM in plantarflexion, they would not satisfy the criteria for somatic dysfunction by restricted ROM.

\section{Data Analysis}

We noted participants whose ankles were restricted or nonrestricted in plantarflexion (compared with the reference values previously noted), and we performed 3 separate data analyses: the whole group, the subgroup of restricted ROM, and the subgroup of nonrestricted ROM.

The online resource QuickCalcs (GraphPad; https:// www.graphpad.com/quickcalcs/ttest1) was used to conduct the statistical analysis. A paired $t$ test was used to analyze the changes in ROM and flutter kick speed. Statistical significance was determined at $P<.05$.

\section{Results}

\section{Demographics}

Of 300 potential participants, 55 were included (response rate, 18.3\%). None of the recruited participants met the exclusion criteria. Fifty-five swimmers (32 females and 23 males; mean age, 12 years) participated in this study. Sixteen participants were in the control group, 17 in the sham group, and 22 in the MET intervention group.

The subgroup with restricted ROM had 42 participants: 12 in the control, 10 in the sham, and 20 in the MET intervention groups. The subgroup with nonrestricted ROM had 13 participants: 4 in the control, 7 in the sham, and 2 in the MET. Table 1 summarizes

\section{Table 1.}

Effects of Post-Isometric Relaxation on Ankle Plantarflexion and Timed Flutter Kick: Demographics of Participants by Group Analysis

\begin{tabular}{clll} 
& \multicolumn{2}{l}{ Gender, No. } & Age, Mean \\
\cline { 2 - 3 } Group & Female & Male & (Range), y \\
\hline Whole Group & & 6 & $12(8-15)$ \\
\hline Control $(n=16)$ & 10 & 7 & $12(8-17)$ \\
\hline Sham $(n=17)$ & 10 & 10 & $12(8-17)$ \\
\hline MET $(n=22)$ & 12 & & \\
\hline Restricted ROM & & 6 & $11(8-15)$ \\
\hline Control $(n=12)$ & 6 & 4 & $13(8-17)$ \\
\hline Sham $(n=10)$ & 6 & 10 & $12(8-16)$ \\
\hline MET $(n=20)$ & 10 & & \\
\hline Nonrestricted ROM & & 0 & $13(12-14)$ \\
\hline Control $(n=4)$ & 4 & 3 & $11(9-13)$ \\
\hline Sham $(n=7)$ & 4 & 0 & $14(11-17)$ \\
\hline MET $(n=2)$ & 2 & &
\end{tabular}

Abbreviation: MET, muscle energy technique; ROM, range of motion. 
Table 2.

Effects of Post-Isometric Relaxation on Ankle Plantarflexion and Timed Flutter Kick in Pediatric Competitive Swimmers: ROM Before and After Intervention by Group

\begin{tabular}{|c|c|c|c|c|c|c|c|c|c|c|}
\hline \multirow[b]{2}{*}{ Group } & \multicolumn{5}{|c|}{ ROM of Left Ankle } & \multicolumn{5}{|c|}{ ROM of Right Ankle } \\
\hline & Before & After & $\Delta^{\mathbf{a}}$ & $\begin{array}{l}P \\
\text { Value }^{\text {b }}\end{array}$ & $95 \% \mathrm{CI}$ & Before & After & $\Delta^{\mathbf{a}}$ & $\begin{array}{l}P \\
\text { Value }^{b}\end{array}$ & $95 \% \mathrm{CI}$ \\
\hline \multicolumn{11}{|l|}{ Whole Group } \\
\hline Control $(n=16)$ & 47.094 & 47.844 & +0.750 & .693 & -4.721 to 3.221 & 46.375 & 46.750 & +0.375 & .796 & -3.404 to 2.654 \\
\hline Sham $(n=17)$ & 52.618 & 50.647 & -1.971 & .382 & -2.675 to 6.616 & 49.324 & 51.294 & +1.97 & .182 & -4.964 to 1.023 \\
\hline $\operatorname{MET}(n=22)$ & 42.659 & 46.568 & +3.909 & .046 & -7.742 to -0.076 & 43.955 & 50.432 & +6.477 & .004 & $\begin{array}{l}-10.690 \text { to } \\
-2.264\end{array}$ \\
\hline
\end{tabular}

\begin{tabular}{|c|c|c|c|c|c|c|c|c|c|c|}
\hline $\begin{array}{l}\text { Restricted ROM } \\
\text { Subgroup }\end{array}$ & & & & & & & & & & \\
\hline Control $(n=12)$ & 43.000 & 44.250 & +1.250 & .594 & -6.263 to 3.763 & 42.292 & 42.542 & +0.250 & .896 & -4.353 to 3.853 \\
\hline Sham $(n=10)$ & 45.300 & 46.700 & +1.400 & .650 & -8.150 to 5.350 & 42.700 & 45.200 & +2.500 & .164 & 1.230 to -6.230 \\
\hline $\operatorname{MET}(n=20)$ & 40.775 & 45.125 & +4.350 & .041 & -8.500 to -0.200 & 42.300 & 48.575 & +6.275 & .011 & $\begin{array}{l}-10.924 \text { to } \\
-1.626\end{array}$ \\
\hline \multicolumn{11}{|l|}{$\begin{array}{l}\text { Nonrestricted } \\
\text { ROM Subgroup }\end{array}$} \\
\hline Control $(n=4)$ & 59.375 & 58.625 & -0.750 & .838 & -9.974 to 11.474 & 58.625 & 59.250 & +0.625 & .680 & -5.001 to 3.751 \\
\hline Sham $(n=7)$ & 63.071 & 56.286 & -6.786 & .027 & 1.085 to 12.486 & 58.786 & 60.000 & +1.214 & .661 & -7.654 to 5.226 \\
\hline $\operatorname{MET}(n=2)$ & 61.500 & 63.00 & +1.500 & .500 & -20.560 to 17.560 & 60.50 & 69.00 & +8.50 & .182 & -40.270 to 23.270 \\
\hline
\end{tabular}

a Mean change in degrees of measured ankle plantarflexion $(\Delta)$ from a standardized starting position in participants before and after intervention (control, sham, or muscle energy technique [MET]).

b $P$ values were calculated using the paired $t$ test.

Abbreviation: ROM, range of motion.

these demographics and provides additional information on gender and age.

\section{ROM of Ankle Plantarflexion}

Table 2 summarizes the change in the ROM of bilateral ankle plantarflexion, as measured before and after the intervention.

Among participants who received MET, the whole group and those in the subgroup of restricted ROM had statistically significant increases in bilateral ankle plantarflexion (whole group: $P=.046$ on the left and $P=.004$ on the right; subgroup of restricted ROM: $P=.041$ on the left and $P=.011$ on the right). Among participants in the control and sham groups, the whole groups and subgroups of restricted ROM did not demonstrate any statistically significant change in plantarflexion from before to after the intervention.

In the subgroup of nonrestricted ROM, there was no significant change in ankle plantarflexion, except in the left ankle of the sham group, which demonstrated significantly less ROM after the sham intervention (a loss of $6.786^{\circ} ; P=.027$ )

\section{Flutter Kick Time}

The change in flutter kick time is summarized in Table 3, as measured before and after the intervention. In both the whole group and the subgroup of restricted ROM, the participants who received MET swam 
Table 3.

Effects of Post-Isometric Relaxation on Ankle Plantarflexion and Timed Flutter Kick in Pediatric Competitive Swimmers: Flutter Kick Swim Time by Group Analysis

\begin{tabular}{|c|c|c|c|c|c|}
\hline & \multicolumn{3}{|c|}{ 25-yard Swim Time, s } & \multirow[b]{2}{*}{$P$ Value $^{\text {b }}$} & \multirow[b]{2}{*}{$95 \% \mathrm{Cl}$} \\
\hline & Before & After & $\Delta^{\mathbf{a}}$ & & \\
\hline \multicolumn{6}{|l|}{ Whole Group } \\
\hline Control $(n=16)$ & 25.269 & 25.196 & -0.073 & 0.843 & -0.848 to 0.702 \\
\hline Sham $(n=17)$ & 22.422 & 22.499 & +0.077 & 0.724 & -0.379 to 0.535 \\
\hline $\operatorname{MET}(n=22)$ & 23.151 & 23.895 & +0.744 & 0.090 & -0.127 to 1.613 \\
\hline \multicolumn{6}{|c|}{ Restricted ROM Subgroup } \\
\hline Control $(n=12)$ & 26.332 & 26.386 & +0.054 & 0.908 & -0.957 to 1.065 \\
\hline Sham $(n=10)$ & 21.939 & 21.674 & -0.265 & 0.251 & -0.753 to 0.223 \\
\hline $\operatorname{MET}(n=20)$ & 23.614 & 24.431 & +0.817 & 0.089 & -0.138 to 1.772 \\
\hline \multicolumn{6}{|c|}{ Unrestricted ROM Subgroup } \\
\hline Control $(n=4)$ & 22.082 & 21.628 & -0.454 & 0.439 & -1.173 to 2.083 \\
\hline Sham $(n=7)$ & 23.111 & 23.679 & +0.568 & 0.172 & -1.462 to 0.327 \\
\hline MET $(n=2)$ & 18.525 & 18.530 & +0.005 & 0.994 & -6.676 to 6.666 \\
\hline
\end{tabular}

a Mean change in speed of flutter kick, measured in seconds over a distance of 25 yards, in participants before and after a randomized intervention (control, sham, or muscle energy technique [MET]).

b $P$ values were calculated with the paired $t$ test.

Abbreviation: ROM, range of motion

slower after the intervention, but the finding was not statistically significant (whole group: $P=.090$; subgroup: $P=.089$ ). There was no significant difference in swimming speed for any of the other groups, including within the subgroup of nonrestricted ROM.

\section{Discussion}

At the outset of this study, we applied an interventional protocol to all participants within randomly assigned groups, blinding the investigators as to whether participants were symptomatic or asymptomatic with regard to restriction of ankle plantarflexion, mitigating the potential bias of treating one participant differently from another. For those participants receiving MET, using the principle of PIR, the technique was applied as a way to gently stretch myofascial structures, to allow for increased plantarflexion, and to observe how this functional gain affects flutter kick performance, which was measured in seconds over 25 yards.

In the clinical setting, the indication for OMT is the diagnosis of somatic dysfunction. We retrospectively diagnosed somatic dysfunction using the most objective criterion-ROM - referenced against a national guideline for normal ROM. ${ }^{15}$ Once we had subgroups of individuals with somatic dysfunction (restricted ROM) and without somatic dysfunction (nonrestricted ROM), we were able to compare these subgroups with each other and with the whole group.

After MET, there was a statistically significant increase in the ROM of both ankle joints in both the whole group and the subgroup of restricted ROM, with no change in the sham or control interventions. These findings supported our hypothesis that MET, using the 
principle of PIR, will significantly improve plantarflexion, notably distinguishing the presence of somatic dysfunction via restricted ROM.

When looking at the raw data points, the 9- and 10 -year-olds (the youngest in the group) were the participants whose left ankles lost ROM (mean, 11.5 ), whereas the older participants (age 13 years) all stayed the same or gained ROM. This finding could be enough to skew the statistics. This loss did not occur on the right side, except in one of the 9-year-old participants. Our speculation is that the younger participants may not have complied properly with the instructions to plantarflex to the comfortable full range when the left ankle was measured (the first ankle of the 2 to be measured in the protocol). Regardless of the reasoning, however, the small subsample provides poor power for this statistical outcome to be meaningful. There was no change, by way of increased plantarflexion, with the control, sham, or MET interventions in the subgroup of nonrestricted ROM. Although this finding may be because these participants' ankles did not have somatic dysfunction because they were not initially restricted in their ROM, it is more likely that the numbers were too small to be powered enough to definitively demonstrate that assertion. In a future study, the numbers in the subgroup of nonrestricted ROM would need to be higher. Looking at the performance data for flutter kick time, in both the whole group and the subgroup of restricted ROM, the participants who received MET were actually slower on postintervention flutter kick than the control or sham groups. However, none of the differences was significant. These findings rejected our hypothesis that MET, using the principle of PIR, will improve flutter kick performance and reduce swim time. Similar results have occurred in studies that observed decreased performance of swimming, jumping, or sprinting when using methods of muscle stretching that differ from PIR - namely, static stretching and proprioceptive neuromuscular facilitation stretching. ${ }^{1,16,17}$

For the subgroup of nonrestricted ROM, the numbers are, again, too small to make any statistical conclusions on swimming performance in participants who have ankle plantarflexion within or above the normal range.

Previous studies have shown that MET is effective at increasing joint ROM. ${ }^{9-11}$ What is less clear is the mechanism by which this increased joint ROM occurs. Traditionally, we speak of a muscle relaxation that occurs after an isometric muscle contraction, whereby there is a refractory period in which the muscle may be passively lengthened without insult to the neuromuscular apparatus. ${ }^{18}$ However, there may be a variety of neurologic and biomechanical mechanisms that affect the change we see with MET. ${ }^{19}$

In the present study, the intervention (control, sham, or MET) was applied directly before the participants were given a physical task (the second timed flutter kick). In the whole group and subgroup of restricted ROM (the groups whose numbers were sufficiently powered to make conclusions), we theorize that the MET participants may have been more fatigued by the PIR, thus slowing them down on the second timed swim. Although we did not evaluate this factor, we retrospectively postulated that high-energy phosphates were depleted with the PIR and that there was not enough recovery time after the intervention to reap the benefit of having increased ROM. Bishop supports a similar theory when addressing warm-up protocols before sports activity. ${ }^{20}$ Future studies will provide a longer recovery period before the second timed swim to test the energy-depletion concept with PIR.

There are a few other notable limitations of this study, some of which may have especially affected performance measures. Beginning with the participants themselves, the age and maturity level of the younger participants seemed to affect their ability to follow instructions without distraction. Furthermore, the participants were at different strength and skill levels with regard to their swimming abilities, which may have affected performance variability. Some participants had already been swimming for close to an hour before being evaluated for the study, which may have affected levels of fatigue, hindering subsequent performance. 
The small sample size in this pilot study limits the generalizability of our findings. A larger study population with participants stratified by age, gender, and skill level would address these potential influences on performance variability.

It would be useful to find participants who have a history of ankle trauma (nonacute sprain/strain, healed fracture, or uncomplicated recovery from ankle surgery), as these participants would be ideal candidates to best benefit from MET. An additional modification of the experimental design could include having separate investigators to document ankle measurements, perform the MET, and measure swim times to eliminate the potential for bias. Because each participant underwent only a single session with a single set of evaluations for ROM and flutter kick time, we are uncertain as to the effects of MET, using PIR, over subsequent days or weeks. Furthermore, other biomechanical factors contribute to swimming performance. In particular, arm strength and ROM are obvious and critical components of swim speed. In our study, the action of the arms was eliminated from the swimming stroke to decrease confounding factors on the effect of ankle ROM. However, controls such as this are not realistic for swimming competitions.

Finally, we chose to use MET's principle of PIR to increase ankle plantarflexion and ROM. Post-isometric relaxation was selected mainly because it can be taught to swimmers, coaches, and trainers. Other types of OMT, such as soft tissue technique and balanced ligamentous tension, can be tested for their effects on ROM and swim time.

\section{Conclusion}

This pilot study reveals that MET, using the principle of PIR, may be a useful tool for improving ankle plantarflexion in swimmers, especially for those who have restricted ROM in their ankles. By removing the aforementioned limitations, future studies will be able to better test the hypothesis that improved plantarflexion enhances the performance of competitive swimmers.

\section{Acknowledgments}

We thank Jane Z. Dumsha, PhD, Chief Research Operations Officer, and Mindy George-Weinstein, PhD, Professor and Chief Research and Science Officer, both at the Philadelphia College of Osteopathic Medicine, for their design input and critical editing of this manuscript.

\section{Author Contributions}

All authors provided substantial contributions to conception and design, acquisition of data, or analysis and interpretation of data; Drs Noto-Bell and Vogel drafted the article or revised it critically for important intellectual content; all authors gave final approval of the version of the article to be published; all authors agree to be accountable for all aspects of the work in ensuring that questions related to the accuracy or integrity of any part of the work are appropriately investigated and resolved.

\section{References}

1. Costa G, Silva E, Silveira A, et al. Acute effects of static and proprioceptive neuromuscular facilitation stretching on sprint performance in male swimmers. Med Sport. 2014;67(1):119-128.

2. Manoel ME, Harris-Love MO, Danoff JV, Miller TA. Acute effects of static, dynamic, and proprioceptive neuromuscular facilitation stretching on muscle power in women. J Strength Cond Res. 2008;22 (5):1528-1534. doi:10.1519/JSC.0b013e31817b0433

3. O'Shea P, O'Shea K, Chay C. The 50-meter freestyle sprint. NSCA Journal. 1991;13(5):9-10.

4. Cureton TK. Mechanics and kinesiology of swimming - the crawl flutter kick. Res Q. 1930;1(4):87-121.

5. Hull M. Flexible ankles: faster swimming. Swimming Technique. 1990;27(3):23-24.

6. Willems TM, Cornelis JA, De Deurwaerder LE, Roelandt F, DeMits S. The effect of ankle muscle strength and flexibility on dolphin kick performance in competitive swimmers. Hum Mov Sci. 2014;36:167-176. doi:10.1016/j.humov.2014.05.004

7. Sifaki O, Zafiriadis S, Tsalis G, Loupos D. Relationship of physical and kinetic characteristics of the ankle joint and the performance of freestyle kick in pre-pubertal swimmers and water polo players. Inquiries Sport Phys Educ. 2014;12(1):9-16.

8. McCullough AS, Kraemer WJ, Volek JS, et al. Factors affecting flutter kicking speed in women who are competitive and recreational swimmers. J Strength Cond Res. 2009;23(7):2130-2136. doi:10.1519/ JSC.0b013e31819ab977

9. Burns DK, Wells MR. Gross range of motion in the cervical spine: the effects of osteopathic muscle energy technique in asymptomatic subjects. J Am Osteopath Assoc. 2006;106(3):137-142.

10. Eisenhart AW, Gaeta TJ, Yens DP. Osteopathic manipulative treatment in the emergency department for patients with acute ankle injuries. J Am Osteopath Assoc. 2003;103(9):417-421.

11. Moore SD, Laudner KG, McLoda TA, Shaffer MA. The immediate effects of muscle energy technique on posterior shoulder tightness: a randomized controlled trial. J Orthop Sports Phys Ther. 2011;41 (6):400-407. doi:10.2519/jospt.2011.3292

12. Keene DJ, Mistry D, Nam J, et al. The Ankle Injury Management (AIM) trial: a pragmatic, multicentre, equivalence randomised controlled trial 
and economic evaluation comparing close contact casting with open surgical reduction and internal fixation in the treatment of unstable ankle fractures in patients aged over 60 years. Health Technol Assess. 2016;20(75):1-158.

13. Willett K, Keene DJ, Morgan L, et al. Ankle Injury Management (AIM) design of a pragmatic multi-centre equivalence randomised controlled trial comparing Close Contact Casting (CCC) to Open surgical Reduction and Internal Fixation (ORIF) in the treatment of unstable ankle fractures in patients over 60 years. BMC Musculoskelet Disord. 2014;15:79. doi:10.1186/1471-2474-15-79

14. Educational Council on Osteopathic Principles. Glossary of Osteopathic Terminology. Bethesda, MD: American Association of College of Osteopathic Medicine; November 2011. http://www.aacom.org/ resources/bookstore/Documents/GOT2011ed.pdf. Accessed July 2018.

15. Soucie JM, Wang C, Forsyth A, et al. Hemophilia Treatment Center Network. Range of motion measurements: reference values and a database for comparison studies. Haemophilia. 2011;17(3):500-507. doi:10.1111/j.1365-2516.2010.02399.x
16. Mikolajec K, Waskiewicz Z, Maszczyk A, Bacik B, Kurek P, Zajac A. Effects of stretching and strength exercises on speed and power abilities in male basketball players. Isokinet Exerc Sci. 2012;20 (1):61-69. doi:10.3233/IES-2012-0442

17. Nelson AG, Driscoll NM, Landin DK, Young MA, Schexnayder IC. Acute effects of passive muscle stretching on sprint performance. J Sport Sci. 2005;23(5):449-454. doi:10.1080/02640410410001730205

18. Mitchell FL Jr, Moran PS, Pruzzo NA. An Evaluation and Treatment Manual of Osteopathic Manipulative Procedure. 2nd ed. Kansas City, MO: Institute for Continuing Education in Osteopathic Principles, Inc; 1973:325.

19. Fryer G. Muscle energy technique: an evidence-informed approach. Int J Osteopath Med. 2011;14(1):3-9. doi:10.1016/j.jjosm.2010.04.004

20. Bishop D. Warm Up II: Performance changes following active warm-up and how to structure warm-up. Sports Med. 2003;33(7):483-498. doi:10.2165/00007256-200333070-00002

() 2019 American Osteopathic Association

\section{Electronic Table of Contents}

More than 135,000 individuals receive electronic tables of contents (eTOCs) for newly posted content to The Journal of the American Osteopathic Association website. To sign up for eTOCs and other JAOA announcements, visit http://jaoa.org/ss/subscribe.aspx. 